EPJ Web of Conferences 81, 01010 (2014)

DOI: $10.1051 /$ epjconf/ 20148101010

(C) Owned by the authors, published by EDP Sciences, 2014

\title{
Encounters with dibaryons - have they finally become true?
}

\author{
H. Clement ${ }^{1,2, a}$, M. Bashkanov ${ }^{1,2}$, E. Perez del Rioo ${ }^{1,2}$, and T. Skorodko ${ }^{1,2,3}$ \\ for the WASA-at-COSY Collaboration
}

${ }^{1}$ Physikalisches Institut, Eberhard-Karls-Universität Tübingen, Auf der Morgenstelle 14, 72076 Tübingen, Germany

${ }^{2}$ Kepler Center for Astro and Particle Physics, University of Tübingen, Auf der Morgenstelle 14, 72076 Tübingen, Germany

${ }^{3}$ Department of Physics, Tomsk State University, 36 Lenina Avenue, 634050 Tomsk, Russia

\begin{abstract}
A short review is given about the long-standing search for dibaryons, i.e. sixquark objects, from the early days until present, when the first non-trivial dibaryon resonance has been established.
\end{abstract}

\section{Introduction}

The long history of dibaryon searches dating back to the fifties has been a very changeful one with many ups and downs. Early predictions of a vast number of dibaryon states initiated endless experimental claims, but finally none survived careful experimental investigations. For a review see, e.g. Refs. [1,2].

Despite their long painful history dibaryon searches have recently received renewed interest, in particular by the recognition that there are more complex quark configurations than just the familiar $q \bar{q}$ and $q q q$ systems - in favor also of hidden-color aspects [3]. Recently two groups announced that lattice QCD calculations [4-7] provide evidence for a bound H-dibaryon - though any experimental evidence is still pending [8].

However, the WASA-at-COSY collaboration has found recently that the double-pionic fusion reaction $p n \rightarrow d \pi^{0} \pi^{0}$ proceeds dominantly via a resonance structure observed in the total cross section at $\sqrt{s}=2.37 \mathrm{GeV}$ with $\Gamma \approx 70 \mathrm{MeV}$ and $I\left(J^{P}\right)=0\left(3^{+}\right)[9]-$ after first signs of it had been observed already at Uppsala by the CELSIUS/WASA collaboration [10]. Meanwhile nearly all possible decay channels have been investigated [11-13], in particular also the one into the elastic $n p$ channel. And, indeed, new data on polarized $n p$ scattering in the region of interest produce a resonance pole in the coupled ${ }^{3} D_{3}-{ }^{3} G_{3}$ partial waves in accordance with the resonance hypothesis $[14,15]$. This gives the first solid evidence that dibaryons really exist.

Since this resonance is observed to decay dominantly via an intermediate $\Delta \Delta$ system, it constitutes asymptotically a $\Delta \Delta$ system bound by nearly $100 \mathrm{MeV}$ - as predicted by Dyson and later-on also by Goldman et al. [16], who called it the "inevitable dibaryon" $d^{*}$ due to its unique symmetry features. Most recent relativistic three-body calculations based on hadron dynamics $[17,18]$ as well as quark model calculations [19-21] succeeded to predict properly a number of characteristics of this

\footnotetext{
a e-mail: heinz.clement@uni-tuebingen.de
} 
resonance. The latter also postulate a substantial hidden-color component accounting in particular for the unusually narrow width of this resonance.

In the following first a short review is given about the early dibaryon searches before we concentrate on the most recent WASA results on this issue.

\section{The early days and the dibaryon rush era}

Early searches for dibaryons date back to the fifties, i.e. far before the substructure of the nucleon was discussed and much before the quark model was introduced. In particular groups at Dubna and Gatchina were looking for resonances in $N N$ scattering and pion production as possible signs of dibaryon states $[22,23]$. Later-on these activities were refined by Dick Arndt and cumulated in the SAID analysis group pursuing state-of-the art partial-wave analyses of $N N, \pi N$ and $\pi d$ scattering.

After Gell-Mann's famous paper [24] on the quark model Dyson \& Xuong [25] were the first to correlate this topic with SU(6) and six quark systems - still in the year 1964. Surprisingly, this first theoretical prediction did not find overwhelming attention by the community - though in retrospect it appears to have predicted remarkably well at least some of the six non-strange candidates, as we will see below.

The real dibaryon rush started in 1977 with Jaffe's paper on a bound $\Lambda \Lambda$ state, the so-called Hdibaryon [26]. This work initiated a copious number of theoretical calculations predicting partly a multitude of dibaryon states, see e.g. Ref. [27]. Animated by the enormous amount of predicted states, a worldwide rush of experimental dibaryon searches set in - ending finally with a vast number of claims. But unfortunately no single one survived critical experimental and analytical examinations.

Among the reasons for this striking failure was certainly the insufficient quality of data, be it lowstatistics bubble-chamber data or data from inclusive measurements, performed often by single-arm detectors. For a critical, though also amusing review of this epoch see, e.g., the one given 1988 by K. $\mathrm{K}$. Seth [1], who pioneered this field by many high-quality measurements unmasking thus many of the dibaryon claims as statistical fluctuations or detector artifacts in poor data. In his conclusions he summarizes:

- "The days of doing quick and dirty (Q \& D) experiments are over."

- "The days of making Q \& D predictions are over."

- "The days of inventing dibaryons to explain the difference between poor experiment and poorer theory (or vice-versa) are over."

- "We must do honest hard work, or quit."

And he recommends for future research: " We should concentrate on exclusive experiments ...", a point, which we will pursue in the following.

\subsection{A remnant from this period - a resonance in the ${ }^{1} D_{2}$ partial wave?}

Possibly, there exists a survivor from this era - a resonance in the ${ }^{1} D_{2}$ partial wave of the $N N$ system. Already in the fifties it was observed $[22,23]$ that this partial wave indicates a resonant behavior in the $\pi^{+} d \rightarrow p p$ reaction corresponding to a resonance with $I\left(J^{P}\right)=1\left(2^{+}\right), \mathrm{m} \approx 2160 \mathrm{MeV}$ and $\Gamma \approx 120 \mathrm{MeV}$. Dyson \& Xuong identified this candidate with their asymptotic $N \Delta$ state to fix the remaining parameter in their ansatz. The possible existence of this resonance was confirmed in the sixties by Dick Arndt [28] analyzing $p p$ scattering data. Later-on, in the eighties and nineties, the SAID data analysis group could demonstrate [29-32] by partial wave anaylses based on high-quality data for $p p \rightarrow p p, \pi d \rightarrow \pi d$ and $\pi d \rightarrow p p$ that the ${ }^{1} D_{2}$ partial wave exhibits a pronounced looping 
in the Argand diagram - in favor of a genuine $s$-channel resonance. However, since the mass of the resonance is close to the $N \Delta$ threshold and since in addition the width of the resonance is compatible with that of the $\Delta$, it has been argued that the observed features represent a $N \Delta$ threshold phenomenon rather than a $s$-channel resonance and the observed looping in the Argand plot is just a reflection of the usual $\Delta$ excitation. In this discussion there have been many pros and cons, see, e.g. Refs. [1,33-37]. In a series of papers $[38,39]$ Hoshizaki concluded that interpretations in terms of a $N \Delta$ virtual-state pole or a threshold cusp are invalid, rather it constitutes a $S$-matrix pole at (2144 - i55) $\mathrm{MeV}$.

But still we are left with the problem that these values do not deviate significantly from what is expected for a conventional $\Delta$ excitation by $t$-channel meson exchange between the two nucleons.

\section{Exclusive and kinematically complete experiments - the first exotic dibaryon resonance}

With the begin of the new millennium the WASA detector with nearly $4 \pi$ angular coverage [40] went into operation at the CELSIUS storage ring at Uppsala, before it was moved later-on to the COSY ring at the research center Jülich [41]. This provided the possibility to measure meson production in $p p$, $p d$ and $d d$ collisions for the first time exclusively and kinematically complete - in most cases even with up to six overconstraints. In addition the high-quality beam ensured a low background situation. That way such measurements delivered data of unprecedented quality.

Since in particular two-pion production was only scarcely investigated experimentally, but had the potential to bear unusual phenomena, a program was set up to measure the various two-pion production channels in $p p$ collisions from threshold up to $T_{p}=1.4 \mathrm{GeV}$. As a result it was shown that all $p p$ induced, i.e. all isovector channels behave regularly as expected from conventional $t$-channel excitation of Roper, $\Delta \Delta$ and $\Delta(1600)$ resonances.[42-48].

The situation changed drastically, when $p n$ initiated two-pion production was looked at. In particular, the purely isoscalar $p n \rightarrow d \pi^{0} \pi^{0}$ reaction, for which no data existed so far, exhibited a pronounced narrow resonance structure in the total cross section - first observed at CELSIUS [10] and later-on at COSY with much improved statistics [9]. Subsequent measurements of all three double-pionic fusion channels, the purely isoscalar $d \pi^{0} \pi^{0}$ channel, the purely isovector $d \pi^{+} \pi^{0}$ channel and the isospin mixed $d \pi^{+} \pi^{-}$channel, clearly showed that this resonance structure is uniquely of isoscalar nature. Fig. 1, left, displays the experimental decomposition of the $d \pi^{+} \pi^{-}$channel (red) in its isoscalar (blue) and isovector (black) components [11]. Note that there is no sign of the resonance observed in the data for the isovector channel, whereas in the isoscalar channel the Lorentzian structure shows up very clearly sitting on top of only a low background due to conventional processes. Hence this channel is suited best for deriving mass and width of this resonance resulting in $\mathrm{m} \approx 2370 \mathrm{MeV}$ and $\Gamma \approx 70 \mathrm{MeV}$ - with these numbers depending somewhat on the treatment of the background. From the angular distributions spin and parity have been determined to be $J^{P}=3^{+}$, whereas the Dalitz plot shows that this resonance decays predominantly via a $\Delta \Delta$ intermediate state, though the resonance mass is about $80 \mathrm{MeV}$ below $2 m_{\Delta}$ [9]. Remarkable is also that its width is more than three times smaller than that of the conventional $t$-channel $\Delta \Delta$ process.

If this isoscalar resonance structure, indeed, represents a genuine $s$-channel resonance, then it has to show up also in $n p$ scattering. Since its effect in the $n p$ observables is expected to be very small [53], the most suited observable appears to be the analyzing power, because it consists only of interference terms making thus this observable particularly sensitive to small contributions. Unfortunately there existed no corresponding data in the energy region of interest. Hence polarized $\vec{n} p$ scattering measurements have been carried out with the WASA setup at COSY [14, 15]. The result is shown on the right-hand side of Fig. 1 for a center-of-mass scattering angle near $90^{\circ}$, where the effect of a 

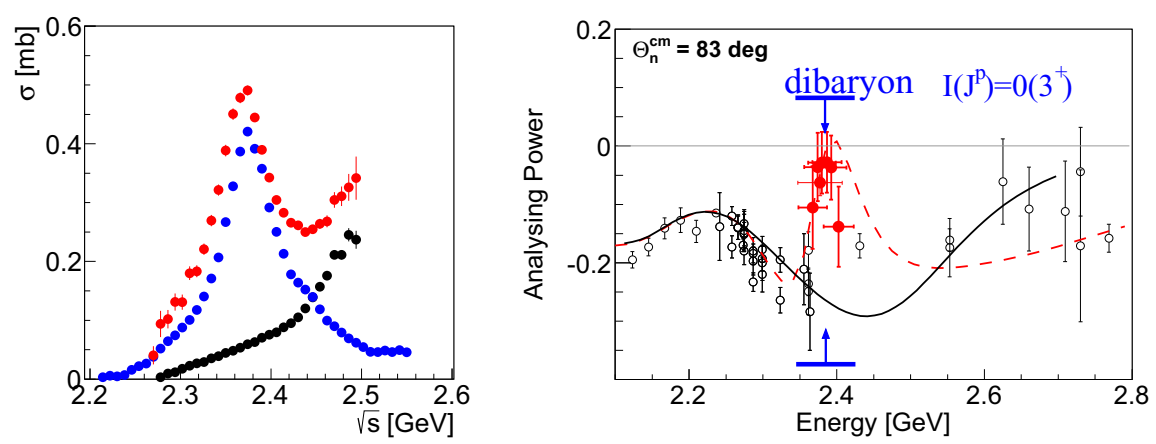

Figure 1. Left: Energy dependence of the total cross section for the double-pionic fusion reaction $p n \rightarrow d \pi^{+} \pi^{-}$ (red) and its isospin decomposition into isoscalar part - corresponding to $2 \sigma\left(p n \rightarrow d \pi^{0} \pi^{0}\right)$ (blue) - and isovector part - corresponding to $1 / 2 \sigma\left(p p \rightarrow d \pi^{+} \pi^{0}\right.$ (black), see Ref. [11]. Right: Energy dependence of the analyzing power in $\vec{n} p$ scattering near $90^{\circ}$, where the effect of a $I\left(J^{P}\right)=0\left(3^{+}\right)$resonance shows up most pronounced. Filled circles denote WASA results, open symbols previous work. The solid line gives the current SAID solution SP07, the dashed line represents the new SAID solution containing the resonance pole $[14,15]$.
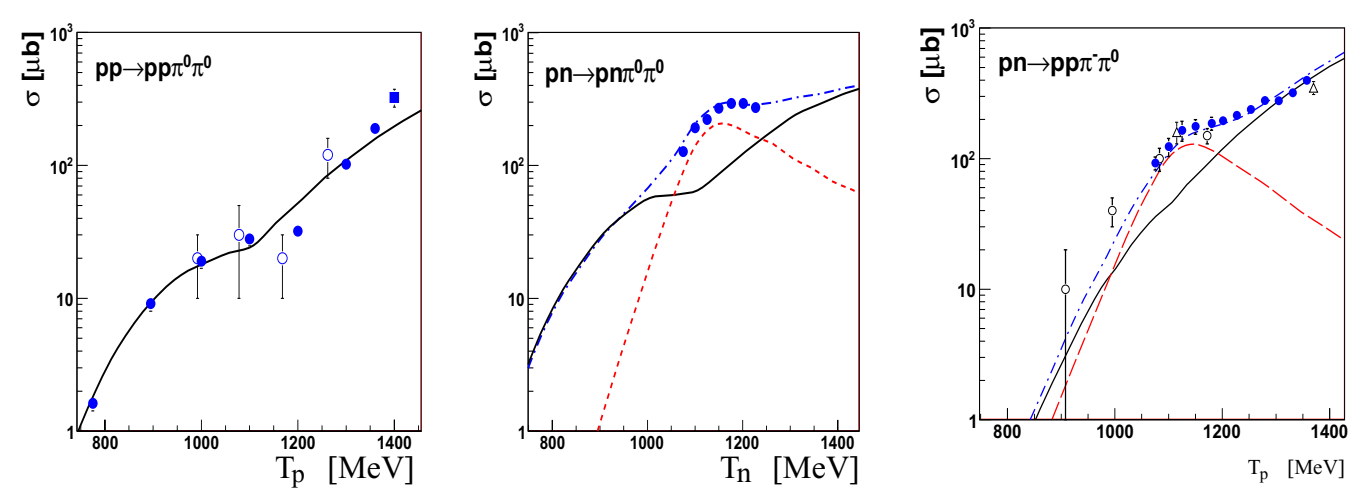

Figure 2. Energy dependence of two-production to the non-fusion channels $p p \rightarrow p p \pi^{0} \pi^{0}$ (left), $p n \rightarrow p n \pi^{0} \pi^{0}$ (middle) and $p n \rightarrow p p \pi^{-} \pi^{0}$ (right). Black, solid lines denote calculations of conventional $t$-channel processes leading to Roper, $\Delta \Delta$ and $\Delta(1600)$ excitations in the modified Valencia model. Red, dashed lines show the $d^{*}$ contributions and blue, dash-dotted lines their coherent sum. Filled blue circles give the WASA data, open symbols previous experimental results. From Refs. [12, 13, 43].

$J^{P}=3^{+}$resonance is largest. The new data (red) exhibit a clear resonance structure in the energy dependence of the analyzing power. Inclusion of the new data in the SAID data base and its subsequent partial-wave analysis produces a pole in the coupled ${ }^{3} D_{3}-{ }^{3} G_{3}$ partial waves at $(2380 \pm 10)-i(40 \pm 5)$ $\mathrm{MeV}$ - in agreement with the resonance hypothesis based on the double-pionic fusion results $[14,15]$ and called now $d^{*}(2380)$ in resemblance of the predicted "inevitable dibaryon".

Furthermore, if this scenario of an $s$-channel resonance decaying via a $\Delta \Delta$ intermediate configuration is correct, then it also should decay into all isospin allowed non-fusion two-pion production 
channels, i.e. the channels $p p \pi^{0} \pi^{-}, p n \pi^{0} \pi^{0}$ and $p n \pi^{+} \pi^{-}$. Since there were no appropriate data, the first two channels were measured with WASA at COSY in the energy region of interest. The WASA results (blue solid dots) for the total cross sections are shown in Fig. 2 together with data from previous experiments (open symbols) [12, 13, 43]. Again, the experimental results are consistent with the appearance of the $d^{*}$ resonance in about the strength expected from isospin relations. Note that since we deal here with a four-body phase-space, which strongly rises with increasing energy, the resonance appears essentially only as a shoulder in the total cross section. However, by comparison to the situation, where this resonance is absent, like in the isovector $p p \pi^{0} \pi^{0}$ channel (Fig. 2, left), the resonance effect in the $p n \pi^{0} \pi^{0}$ and $p p \pi^{0} \pi^{-}$channels appears to be obvious.

For the $p n \pi^{+} \pi^{-}$channel there exist at present only bubble-chamber data, which appear to be consistent with the resonance hypothesis within their very limited precision. However, high-quality data may soon get available, since the HADES collaboration has performed measurements for this channel in the energy region of interest.

\section{Summary and outlook}

After a vast number of unsuccessful searches a non-trival dibaryon resonance has now been found and its major decay channels identified. What is missing, is a measurement of its electromagnetic form factor, in order to learn about the size of this object - whether it is of molecular type or a compact six-quark entity.

New state-of-the-art three-body [17, 18] and quark-model [19-21] calculations reproduce a number of characteristics of this resonance. Interestingly, also the first prediction for the mass of this resonance, the one by Dyson \& Xuong, turns out to be already surprisingly close to the experimental result.

Dyson \& Xuong as well as the new generation of theoretical calculations predict a few further very interesting dibaryon resonances, in particular a $I\left(J^{P}\right)=3\left(0^{+}\right)$state, as discussed in some detail in Ref. [3]. Is it just a matter of time, until those are discovered, too?

\section{Acknowledgements}

We acknowledge valuable discussions with J. Haidenbauer, C. Hanhart, A. Kacharava, E. Oset, I. Strakovsky, C. Wilkin and R. Workman on this issue. This work has been supported by BMBF, Research Center Jülich (COSY-FFE) and DFG (CL 214/3-1).

\section{References}

[1] K. K. Seth, Proc. BARYON-BARYON INTERACTION AND DIBARYONIC SYSTEMS, Bad Honnef 1988, 41 (1988)

[2] K. K. Seth, Few-Body Systems 45, 85 (2009); arxiv:0906.1558 [hep-ex].

[3] M. Bashkanov, S. Brodsky and H. Clement, Phys. Lett. B727, 438 (2013).

[4] S. R. Beane et al., Phys. Rev. Lett.106, 162001 (2011).

[5] T. Inoue et al., Phys. Rev. Lett.106, 162002 (2011).

[6] S. R. Beane et al., Phys. Rev. D87, 034506 (2013).

[7] T. Inoue arXiv:hep-lat 1212.4230

[8] B. H. Kim et al. (Belle Collaboration) Phys. Rev. Lett 110, 222002 (2013).

[9] P. Adlarson et al., Phys. Rev. Lett. 106, 242302 (2011). 
[10] M. Bashkanov et al., Phys. Rev. Lett. 102, 052301 (2009).

[11] P. Adlarson et al., Phys. Lett. B 721, 229 (2013).

[12] P. Adlarson et al., Phys. Rev. C 88, 055208 (2013).

[13] P. Adlarson et al., arxiv:1409.2659 [nucl-ex].

[14] P. Adlarson et al., Phys. Rev. Lett. 112, 202301 (2014).

[15] P. Adlarson et al. Phys. Rev. C 90, 035204 (2014).

[16] T. Goldman et al., Phys. Rev. C 39, 1889 (1989).

[17] A. Gal and H. Garcilazo, Phys. Rev. Lett. 111, 172301 (2013).

[18] A. Gal and H. Garcilazo, Nucl. Phys. A 928, 73 (2014).

[19] H. Huang, J. Ping and F. Wang, Phys. Rev. C89, 034001 (2014).

[20] X. Q. Yuan, Z. Y. Zhang, Y. W. Yu and P. N. Shen, Phys. Rev. C. 60, 045203 (1999).

[21] F. Huang, Z. Y. Zhang, P. N. Shen and W. L. Wang, arxiv:1408.0458 [nucl-th].

[22] B. S. Neganov and L. B. Parfenov, Soviet Phys.-JETP 7, 528 (1958).

[23] M. G. Meshcheriakov and B. S. Neganov, Dokl. Akad. Nauk SSSR 100, 677 (1955)

[24] M. Gell-Mann, Phys. Lett. B 8, 214 (1964).

[25] F. J. Dyson and N.-H. Xuong, Phys. Rev. Lett. 13, 815 (1964).

[26] R. L. Jaffe, Phys. Rev. Lett. 38, 195 and 617(E) (1977).

[27] P. J. Mulders, A. T. Aerts and J. J. de Swart, Phys. Rev. D 21, 2653 (1980).

[28] R. A. Arndt, Phys. Rev. 165, 1834 (1968).

[29] R. Bhandari, R. A. Arndt, L. D. Roper and B. J. VerWest, Phys. Rev. Lett. 46, 1111 (1981).

[30] R. A. Arndt, I. Strakovsky, R. L. Workman and D. V. Bugg, Phys. Rev. C 48, 1926 (1993).

[31] R. A. Arndt, I. Strakovsky and R. L. Workman, Phys. Rev. C 50, 1796 (1994).

[32] C. H. Oh, R. A. Arndt, I. Strakovsky and R. L. Workman, Phys. Rev. C 56, 635 (1997).

[33] R. L. Shypit et al., Phys. Rev. Lett. 60, 901 (1988).

[34] R. L. Shypit et al., Phys. Rev. C 40, 2203 (1989).

[35] V. V. Anisovich, A. V. Sarantsev and D. V. Bugg, Nucl. Phys. A 537, 501 (1992).

[36] M. G. Ryskin and I. I. Strakovsky, Phys. Rev. Lett. 61, 2384 (1988).

[37] I. I. Strakovsky, Sov. J. Part. Nucl. 22, 296 (1991).

[38] N. Hoshizaki, Phys. Rev. C 45, R1414 (1992).

[39] N. Hoshizaki, Prog. Theor. Phys. 89, 245, ibid. 251, 563, 569 (1993).

[40] Chr. Bargholtz et al., Nucl. Inst. Meth. A 594, 339 (2008).

[41] H. H. Adam et al., arxiv: nucl-ex/0411038.

[42] F. Kren et al.,Phys. Lett. B 684, 110 (2010) and B 702, 312 (2011); arXiv:0910.0995v2 [nucl-ex]

[43] T. Skorodko et al., Phys. Lett. B 695, 115 (2011).

[44] T. Skorodko et al.,Eur. Phys. J. A 47, 108 (2011).

[45] T. Skorodko et al.,Phys. Lett. B 679, 30 (2009).

[46] P. Adlarson et al., Phys. Lett. B 706, 256 (2011).

[47] S. Abd El-Bary et al., Eur. Phys. J. A 37, 267 (2008).

[48] S. Abd El-Samad et al., Eur. Phys. J. A 42, 159 (2009).

[49] J. Johanson et al., Nucl. Phys. A 712, 75 (2002).

[50] W. Brodowski et al., Phys. Rev. Lett. 88, 192301 (2002).

[51] J. Pätzold et al.,Phys. Rev. C 67, 052202 (2003).

[52] T. Skorodko et al.,Eur. Phys. J. A 35, 317 (2008).

[53] A. Pricking, M. Bashkanov and H. Clement, arxiv:1310.5532 [nucl-ex]. 